\title{
A Topical Preservative-free Fixed-dose Combination Remedy in Patients with Glaucoma with Insufficient Response to Monotherapy - The VISIONARY Study
}

An Expert Interview with Francesco Oddone

IRCCS Fondazione G.B. Bietti, Rome, Italy

DOI: https://doi.org/10.17925/EOR.2019.13.2.65

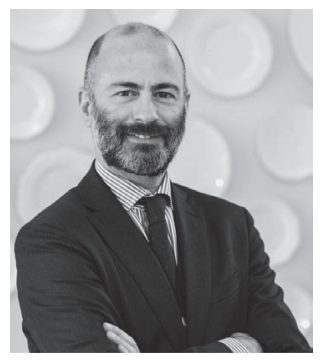

\section{Francesco Oddone}

Francesco Oddone was born in Rome and graduated with honours in Medicine and Surgery from the University of Rome - Tor Vergata where he also specialised with honours in Ophthalmology. He obtained his PhD in 2007 from the same university with a study on the genetic variability of pigment dispersion syndrome and pigmentary glaucoma. He served as a clinical and research fellowship in the Glaucoma Unit of Moorfields Eye Hospital in London, where began scientific collaborations focusing on cutting-edge strategies for the early diagnosis of glaucoma and its monitoring over time. He performs clinical, surgical and scientific activities at the IRCCS Fondazione G.B. Bietti since 2004, and from 2015 held the position of Head of the Glaucoma Unit.

\section{Keywords}

Fixed dose combination, open-angle glaucoma, beta-blocker, prostaglandin analogue

Disclosure: Francesco Oddone has received consultancy fees from Santen, Allergan and Novartis.

Acknowledgement: Medical writing assistance was provided by Katrina Mountfort of Touch Medical Media, and supported by Touch Medical Media.

Review Process: This is an expert interview and, as such, has not undergone the journal's standard peer review process.

Compliance with Ethics: This is an expert interview and did not involve any studies with human or animal subjects performed by any of the authors.

Authorship: The named author meets the International Committee of Medical Journal Editors (ICMJE) criteria for authorship of this manuscript, takes responsibility for the integrity of the work as a whole, and has given final approval to the version to be published.

Received: 6 November 2019

Published Online: 20 December 2019

Citation: European Ophthalmic Review.

2019;13(2): Epub ahead of print

Corresponding Author: Francesco Oddone, Head of the Glaucoma Unit, IRCCS Fondazione G.B.Bietti, Rome, Italy. E: oddonef@gmail.com

Support: No funding was received in

the publication of this article.
G laucoma is a progressive optic neuropathy and is the leading cause of irreversible blindness worldwide. ${ }^{1}$ Despite substantial improvements in technology, therapies, and our understanding of the disease, people continue to go blind from glaucoma due to inadequate disease control. ${ }^{1}$ Medical treatment is initiated with a single topical hypotensive agent, as recommended by the European Glaucoma Society. ${ }^{2}$ However, monotherapy may be insufficient in many patients, because target pressure is not achieved and/or glaucoma progression cannot be prevented by use of a single agent. In these cases, combination therapy is needed. ${ }^{2} \mathrm{~A}$ combination of drugs from different classes with distinct mechanism of actions can be administered either as two separate medications or as a fixed combination. The latter has advantages as they require fewer drops to be applied and thus may improve adherence, and decrease exposure to preservatives. The fixed combination of tafluprost $0.0015 \%$ and timolol $0.5 \%$ (TAF/TIM FC) is preservative free, avoiding the irritation, and other ocular symptoms, associated with preservatives. ${ }^{3}$ This combination has been found to be equivalent to the nonfixed combination of tafluprost $0.0015 \%$ and timolol $0.5 \%$ dosed concomitantly in terms of intraocular pressure (IOP) control and safety. ${ }^{4}$ It has also been shown to be superior to each of its individual preservative-free components. ${ }^{5}$

At this year's European Society of Ophthalmology (SOE) congress, which was held on 13-16 June 2019, in Nice, France, data were released from the VISIONARY study. This was a multicentre, European prospective study that evaluated the effectiveness, tolerability and safety of TAF/TIM FC in routine clinical practice in adults with open-angle glaucoma (OAG) and ocular hypertension who demonstrated an insufficient response to previous monotherapy treatment with topical beta blockers or prostaglandin analogues. ${ }^{6}$ In an expert interview, Dr Oddone discusses the findings of the VISIONARY study.

\section{Q. In open angle glaucoma, how much of a problem is insufficient response to monotherapy with beta blockers or prostaglandin analogues - is effective treatment for this group a substantial unmet need?}

An insufficient response to any hypotensive therapy in glaucoma can be defined as an intraocular IOP above a pre-defined target or as an evidence of disease progression despite the pre-defined target having been reached, indicating that a lower target is required to control the disease. To answer the question about how much this is a problem in patients treated with either beta blockers or prostaglandin analogues, we can refer to the results of major randomised controlled trials (RCTS). For example, the United Kingdom Glaucoma Treatment Study showed us that $15.2 \%$ of patients 
may show evidence of visual field progression when initially treated with a prostaglandin analogue monotherapy. In terms of beta-blockers, in the Low Pressure Glaucoma Treatment Study 39.2\% of patients treated with beta-blocker monotherapy showed evidence of visual field progression, ${ }^{8}$ indicating the necessity for intensification of the hypotensive therapy to control the disease. Moreover, in the Collaborative Initial Glaucoma Treatment Study, where a demanding individualised target IOP was set, within 2 years of enrolment, $75 \%$ of the patient population required more than one hypotensive drug to reach that target. ${ }^{9}$

Based on these data, and on the findings from daily clinical practice, insufficient response to monotherapy with beta blockers or prostaglandin analogues is an issue for a significant proportion of patients at an early or later stage of their disease, and thus an effective and convenient treatment after monotherapy is needed.

\section{Q. The VISIONARY study used a fixed- dose combination of topical tafluprost $(0.0015 \%)$ and timolol $(0.5 \%)$ to treat open angle glaucoma in patients who showed insufficient response to monotherapy. What was the rationale behind the study design and is this a novel treatment combination for such patients?}

Where monotherapies do not control the disease, fixed combination therapy may provide several additional benefits such as improved IOP control, reduced number of daily administrations and reduced exposure to preservatives. TAF/TIM FC has been shown to be efficacious and well tolerated in phase III RCTs and in diverse real-world settings over a 4-16 week period. ${ }^{45,10-12}$ The VISIONARY study investigated its effectiveness and tolerability in a broad population with an insufficient response to either beta blockers or prostaglandin analogues monotherapy over a longer period of time. ${ }^{6}$

\section{Q. Why did the VISIONARY study use a 'real-} world' population of patients with open angle glaucoma in routine clinical practice? Have there been other clinical trials on this drug combination in other patient populations with open angle glaucoma?

As mentioned before, the explanatory RCTs performed for regulatory purposes demonstrated both the superiority of this fixed combination compared to the individual components and the equivalence compared to their concomitant use. ${ }^{4,5}$ Nevertheless, although RCTs remain the gold standard approach for robust assessment of efficacy and safety for new medicines undergoing regulatory approval, observational studies may provide complementary insights concerning those patients that ophthalmologists are most likely to see in clinic. For example, these patients may present with varying degrees of relevant comorbidities that might exclude them from participation in traditional RCTs. In addition, observational studies reflect real-world practice regarding treatment switch, where patients may be changed to a new topical glaucoma medication or formulation without prior treatment washout. Moreover, large-scale observational studies may provide relevant information regarding safety issues that may be not provided by regulatory RCTs. These aspects of observational studies broaden the evidence base for existing treatments, providing important and relevant data that may support ophthalmologists in decision making in regard to therapeutic options.

\section{Q. Preliminary results from the VISIONARY study were released at European Society of Ophthalmology 2019; what were the key efficacy findings?}

The preliminary results of the VISIONARY study presented at the SOE congress in 2019 showed that TAF/TIM FC provided an overall additional $25.3 \%$ reduction of IOP in patients treated with either beta-blocker or prostaglandin analogue monotherapies. I think this represents an interesting finding that may contribute to the process of decision making in clinical practice.

\section{Q. What were the key preliminary safety/ tolerability findings from the VISIONARY study?}

The most interesting finding was a $43.6 \%$ reduction in conjunctival hyperaemia compared to previously used therapies. Moreover, more than one-third of the patient population experienced improvements in dry eye symptoms such as irritation and foreign body sensations. These tolerability findings are particularly relevant if we remember that the ultimate goal of any glaucoma treatment is to protect the quality of life of the patient which could be primarily compromised by the side effects of topical drugs.

\section{Q. Given the VISIONARY study findings, are combinations, such as topical tafluprost and timolol, likely to be the future of open angle glaucoma treatment in patients who show insufficient response to monotherapy?}

The VISIONARY study showed that in real-world clinical practice, that TAF/ TIM FC may provide significant IOP reductions in patients with OAG and ocular hypertension who are insufficiently controlled on prostaglandin analogues or beta-blocker monotherapy. Based on these findings, we can say that prostaglandin analogue fixed combinations, such as TAF/TIM $\mathrm{FC}$, may represent an efficient choice to control IOP in patients showing an insufficient response to monotherapies. $\square$
Tham YC, Li X, Wong TY, et al. Global prevalence of glaucoma and projections of glaucoma burden through 2040: a systematic review and meta-analysis. Ophthalmology. 2014;121:2081-90

2. European Glaucoma Society Terminology and Guidelines for Glaucoma, 4th Edition - Chapter 3: Treatment principles and options Supported by the EGS Foundation: Part 1: Foreword: Introduction; Glossary; Chapter 3 Treatment principles and options. Br J Ophthalmol. 2017:101:130-95.

3. Jaenen N, Baudouin C, Pouliquen P, et al. Ocular symptoms and signs with preserved and preservative-free glaucoma medications. Eur J Ophthalmol. 2007;17:341-9.

4. Hollo G, Hommer A, Anton Lopez A, et al. Efficacy, safety, and tolerability of preservative-free fixed combination of and tolerability of presenative free fixed combination of tafluprost $0.0015 \%$ /timolol $0.5 \%$ versus concomitant use Pfeiffer N, Traverso CE, Lorenz K, et al. A 6-month study comparing efficacy, safety, and tolerability of the preservativefree fixed combination of tafluprost $0.0015 \%$ and timolol $0.5 \%$ versus each of its individual preservative-free components. Adv Ther. 2014;31:1228-46.

6. Oddone F. Treatment of open-angle glaucoma and ocular hypertension withpreservative-free tafluprost/timolol fixeddose combination therapy: interim analysis (12-weeks) of reaworld data (the VISIONARY study). Presented at the European Society of Ophthalmology (SOE) congress. 13-16 June 2019, Nice, France Abstract EP-GLA-06.

Garway-Heath DF, Crabb DP, Bunce C, et al. Latanoprost for open-angle glauComa (UKGTS): a randomised, multicentre, paran

Krupin T, Liebmann JM, Greenfield DS, et al. The Low-pressure Glaucoma Treatment Study (LOGTS) study design and baseline characteristics of enrolled patients. Ophthalmology. 2005;112:376-85.
9. Musch DC, Gillespie BW, Lichter PR, et al. Visual field progression in the Collaborative Initial Glaucoma Treatment Study the impact of treatment and other baseline factors. Ophthalmology. 2009;116:200-7.

10. Suzuki K, Otsuka N, Hizaki H, et al. Multicenter, randomized controlled study comparing tafluprost/timolol fixed combination with latanoprost/timolol fixed combination in primary open-angle glaucoma and ocular hypertension. Adv Ther. 2018;35:796-808.

11. Hollo G, Vuorinen J, Tuominen J, et al. Fixed-dose combination of tafluprost and timolol in the treatment of open-angle glaucoma and ocular hypertension: comparison with other fixed-combination products. Adv Ther 2014:31:032-44.

Pillunat E, Exb C, Ropo A, et at. Preservative free fixed Combination of tafluprost $0.0015 \%$ and timolol $0.5 \%$ in patients
contlonat LE, Erb C, Ropo A, al. Preservative-free fixed with with open-angle glaucoma and ocular hypertension. results of an open-label observational study. Clin Ophthalmol. 2017;11:1051-64. 